\title{
Tunnel Contacts for Spin Injection into Silicon: The Si-Co Interface with and without a MgO Tunnel Barrier-A Study by High-Resolution Rutherford Backscattering
}

\author{
S. P. Dash, ${ }^{1,2}$ D. Goll,, ${ }^{2,3}$ P. Kopold, ${ }^{2}$ and H. D. Carstanjen ${ }^{2}$ \\ ${ }^{1}$ Department of Microtechnology and Nanoscience, Chalmers University of Technology, 41296 Göteborg, Sweden \\ ${ }^{2}$ Max Planck Institute for Intelligent Systems (Formerly Max Planck Institute for Metals Research), Heisenbergstrße. 3 , \\ 70569 Stuttgart, Germany \\ ${ }^{3}$ Materials Research Institute, Aalen University, Beethovenstraße. 1, 73430 Aalen, Germany \\ Correspondence should be addressed to S. P. Dash, saroj.dash@chalmers.se
}

Received 30 June 2011; Revised 20 September 2011; Accepted 20 September 2011

Academic Editor: Adam Georg Balogh

Copyright ( $) 2012$ S. P. Dash et al. This is an open access article distributed under the Creative Commons Attribution License, which permits unrestricted use, distribution, and reproduction in any medium, provided the original work is properly cited.

\begin{abstract}
In order to obtain high spin injection efficiency, a ferromagnet-semiconducor Schottky contact must be of high crystalline quality. This is particularly important in the case of ferromagnet-silicon interfaces, since these elements tend to mix and form silicides. In this study Co-Si (100) interfaces were prepared in three different ways: by evaporation at room temperature, low temperature $\left(-60^{\circ} \mathrm{C}\right)$, and with $\mathrm{Sb}$ as surfactant, and their interface structures were analyzed by high-resolution RBS (HRBS). In all cases more or less strong in-diffusion of Co with subsequent silicide formation was observed. In order to prevent the mixing of Co and $\mathrm{Si}$, ultra thin $\mathrm{MgO}$ tunnel barriers were introduced in-between them. In situ HRBS characterization confirms that the MgO films were very uniform and prevented the mixing of the Si substrate with deposited Co and Fe films effectively, even at $450^{\circ} \mathrm{C}$.
\end{abstract}

\section{Introduction}

Exploiting the spin of the electron in addition to its charge to explore a new generation of spintronic devices which will be smaller, more versatile and more robust than those currently making up silicon chips have both fundamental and technological importance [1-7]. High spin polarization of Co at room temperature $(\sim 40 \%)[8]$ and expected long spin coherence length in $\mathrm{Si}$ (longer than micrometers) [9] make the material couple $\mathrm{Co}$ and $\mathrm{Si}$ attractive for spin injection experiments. In such a heterostructure of a Co thin film on a Si substrate, any structural disorder at the interface would drastically reduce the spin polarization at the interface and, hence, the spin injection efficiency [10-15]. If a small amount of Co diffuses into the $\mathrm{Si}$, each such Co atom will be likely to carry a local magnetic moment oriented randomly with respect to the magnetization direction of the Co thin film. They will scatter the injected spin polarized electrons, thereby degrading their spin polarization $[14,15]$.

Co and Si are known to form various types of silicides that exhibit different degrees of magnetization, that is, nonmagnetic, paramagnetic, or ferromagnetic. When spinpolarized electrons pass through such a silicide region, their polarization is very likely to degrade. The processes involved have not been treated completely and in detail up to present, but the following effects may play an important role: electrons enter a region of different magnetization (magnitude or orientation). Electrons pass a rough interface between regions of different magnetization; such rough interfaces are known to cause strong depolarization [10]. In paramagnetic areas the magnetic moments commonly are not oriented; similarly to the above case of isolated Co atoms they will scatter the injected electrons. In a similar way any kind of magnetic defect may work. One also should not forget about the depolarizing influence of the various Schottky contacts between different silicides.

Thus in order to control and improve the properties of the interface, a detailed understanding of its structure is necessary. In this paper we present results where we studied the Co-Si (100) interface prepared in four different ways: (1) by deposition of $\mathrm{Co}$ at room temperature, (2) by deposition of $\mathrm{Co}$ at low temperature $\left(-60^{\circ} \mathrm{C}\right),(3)$ by deposition of $\mathrm{Co}$ 
at room temperature using $\mathrm{Sb}$ as surfactant, and (4) with a $\mathrm{MgO}$ tunnel barrier at the Co-Si interface. The different types of interfaces were analyzed in situ, that is, during preparation by high depth resolution Rutherford backscattering spectroscopy (HRBS). The HRBS data in turn provide depth profiles of $\mathrm{Co}, \mathrm{Si}, \mathrm{Sb}, \mathrm{Mg}$, and $\mathrm{O}$ with monolayer depth resolution. In this way they give information about the sharpness of the interface and the homogeneity of the Co and $\mathrm{MgO}$ thin films and allow in particular identifying various types of cobalt silicides as a function of depth. The data on the Co-Si Schottky interface have been published before in three publications [16-18] and are here summarized in a comparative way. The data on the $\mathrm{MgO}$ tunnel barrier are new.

\section{The Direct Co-Si (100) (Schottky) Interface}

2.1. Introduction. The phenomena observed at the initial stages of Co deposition on a Si substrate seem to be unique and depend on the preparation conditions and are still discussed controversially [19-23]. About the growth mode of Co on Si, Cho et al. [19] suggested that the Co atoms grow in a layer-by-layer mode, without any interdiffusion, whereas Meyerheim et al. [20] and Rangelov et al. [21] could see an in-diffusion of Co atoms for coverages higher than $0.5 \mathrm{ML}$ of Co. For the Si (100) surface, AES and surface-extended Xray-absorption fine-structure (EXAFS) measurements [1923 ] showed that one can distinguish between several stages of the initial adsorption of Co on Si (100) surfaces at room temperature. About the atomic positions of Co atoms on Si (100) (see Figure 1), Meyerheim et al. [20], Scheuch et al. [22], and Gomoyunova et al. [23] found that Co is adsorbed in fourfold hollow sites (nearly in plane, $\mathrm{d} \approx 0$ ) in every second (110) row of the $\mathrm{Si}$ (100) surface. Cho et al. [19] have found that the preferred adsorption sites are on top of a $\mathrm{Si}$ dimer ( $T_{4}$ sites) and sites spanning the (110) trench (HB sites). However, density functional calculations [24] suggested that the $T_{4}$ and $\mathrm{HB}$ sites are energetically unfavourable (see Figure 1). Concerning the positions of the in-diffused Co atoms, Meyerheim et al. [20] could distinguish between several stages of growth. In the regime of $0.5-2.5 \mathrm{ML}$, Co atoms diffuse into the Si lattice occupying interstitial sites, for the regime above $2.5 \mathrm{ML}$ the substitution of the Si host atoms by Co takes place, and for coverages above $19 \mathrm{ML}$ a locally ordered metallic overlayer starts to grow.

Controversies also exist about the growth mode of Co. Does there exist a critical coverage of Co for the in-diffusion of $\mathrm{Co}$ and the out-diffusion of Si atoms? How thick is the silicide layer formed at the interface, what is its chemical composition, and which are the diffusing species at different stages of growth?

In a previous letter [16] we have addressed some of these controversies by an in situ investigation of the growth of thin Co films (0.08-2.93 ML) on $\mathrm{Si}(100)$ at room temperature with high-resolution Rutherford backscattering spectrometry (HRBS). It turned out that Co diffuses into the Si bulk right from the beginning, that is, already at a Co coverage as low as $0.08 \mathrm{ML}$. For higher coverage various silicide phases start to grow at the surface, but no pure Co film, even at the highest coverage $(2.93 \mathrm{ML}$ of $\mathrm{Co})$ is investigated in this study. In order to reduce the in-diffusion of Co and the outdiffusion of $\mathrm{Si}$ and to enhance the growth of layers of pure Co two ways were followed in two subsequent investigations: (a) Co deposition was done while keeping the substrate at lower temperature, $-60^{\circ} \mathrm{C}[17]$; (b) a thin layer of surfactant (1 ML of $\mathrm{Sb}$ ) was used to reduce the amount of out-diffusion of $\mathrm{Si}$ to the surface during Co deposition [18]. Both techniques were helpful to reduce the silicide formation to some extent but could not stop it in total. The results of these three investigations are reported in a summarized version in the subsequent Sections 2.2 and 2.3.

2.2. Experimental. The in situ HRBS measurements were performed in an ultra high vacuum (UHV) system consisting of a preparation chamber, connected to a Pelletron accelerator, and an electrostatic spectrometer [25] for energy analysis of the scattered ions. In case of room temperature (RT) and low-temperature (LT) deposition of $\mathrm{Co}, 2 \mathrm{MeV} \mathrm{N}^{+}$ ions at incidence angles of $2^{\circ}$ to the sample surface and at a scattering angle of $37.5^{\circ}$ were used for the HRBS analysis. The energy resolution of the spectrometer setup was $4 \mathrm{keV}$ in this case which corresponds to $1 \AA$ depth resolution in $\mathrm{Si}$ and $0.5 \AA$ in Co. In two cases (surfactantmediated deposition and RT deposition of higher amounts of Co, Figure 4) $2 \mathrm{MeV} \mathrm{He}^{+}$ions were used for analysis with incidence angles of $7.5^{\circ}$ and $4.5^{\circ}$, respectively, but the same scattering angle of $37.5^{\circ}$. According to this scattering angle all analysis experiments presented here are actually "forward scattering experiments", but from principle forward and backscattering are all Rutherford scattering. And since Rutherford backscattering spectroscopy (RBS) is the far better known technique, we will call it HRBS in the following. We also want to note that, in order to minimize effects of radiation damage due to the ion beam, a new beam spot on the Si wafer was used for each measurement. Control spectra was taken on the same spot to estimate the influence of radiation damage and potential mixing, but within statistical errors no effect was found.

For the deposition experiments chemically cleaned nSi (100) with resistivity of $4-10 \Omega \mathrm{cm}$ (P doped) were used which were further cleaned in UHV by flash heating at $950^{\circ} \mathrm{C}$. From this temperature the samples were slowly cooled down to RT or $-60^{\circ} \mathrm{C}$, depending on the experimental requirement. These temperatures were maintained as well during Co deposition as during the HRBS measurements. The surface cleanness of the Si samples was verified by HRBS measurements (sensitivity about $5 \%$ ). Co with $4 \mathrm{~N}$ purity was evaporated from an effusion cell at a rate of $0.05 \mathrm{ML} / \mathrm{min}$ $\left(1 \mathrm{ML}=6.87 \times 10^{14}\right.$ atoms $/ \mathrm{cm}^{2}=$ number density of $\mathrm{Si}$ (100) layers). Before use the effusion cell was outgassed; no $\mathrm{C}$ or $\mathrm{O}$ contaminants were found during evaporation. The evaporation rate was calibrated by HRBS with an accuracy of about $5 \%$. As mentioned above, each HRBS spectrum was taken on a new spot $\left(\right.$ size $\left.\sim 1 \mathrm{~mm}^{2}\right)$ to minimize the influence of radiation damage. In the case of surfactantmediated growth of Co, $1 \mathrm{ML}$ of Sb was deposited before the evaporation of Co. This monolayer of $\mathrm{Sb}$ was supposed to 

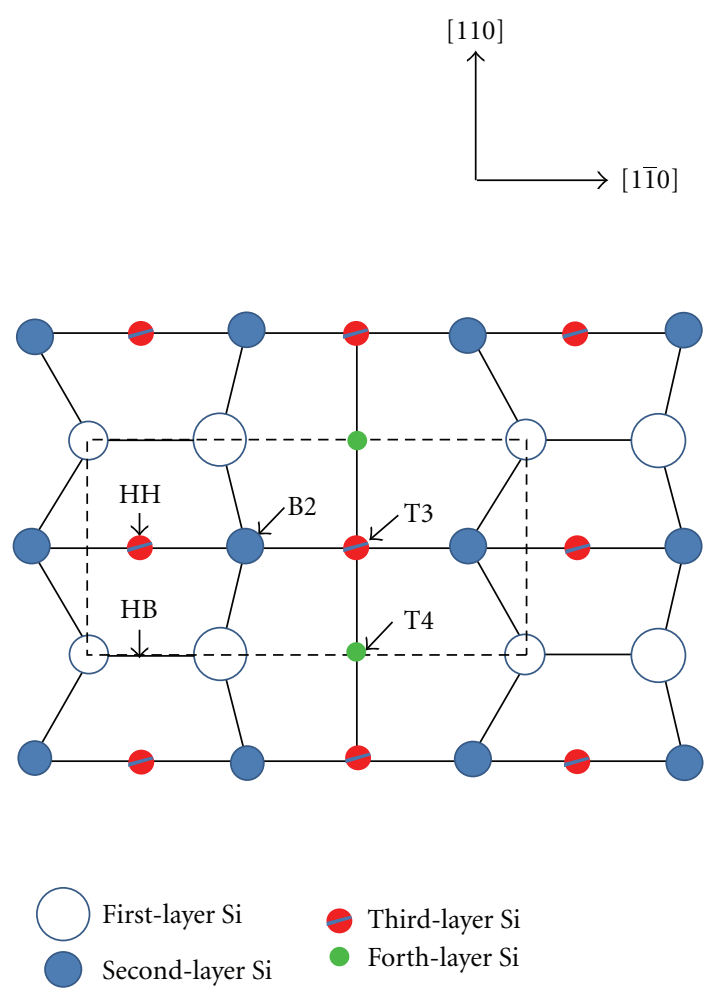

Third-layer Si

Forth-layer Si

Figure 1: The initial Co adsorption sites T4, HB, HH, and T3 on the Si $(100)-(2 \times 1)$ surface at room temperature. B2 is the "cave site" for Mg mentioned in Section 3.3.

float at the surface of the deposited Co, in this way preventing the outdiffusion of Si.

2.3. Results and Discussion. Figures 2(a) and 2(c) show HRBS spectra of Co on Si (100) during the deposition of small amounts of Co (less than $3 \mathrm{ML}$ ) for the substrate temperatures of $20^{\circ} \mathrm{C}$ and $-60^{\circ} \mathrm{C}$, respectively. In addition, Co depth profiles are shown in Figures 2(b) and 2(d) as obtained from the spectra by simulating the spectra with the computer code RUMP [26]. In these simulations the Si sample was subdivided into monolayers of Si (each $6.87 \times$ $10^{14}$ atoms $/ \mathrm{cm}^{2}$ thick) and the Co concentration in each layer adjusted until an optimum fit between simulation and HRBS spectrum was obtained. As is obvious from the spectra, Co atoms diffuse into the Si sample right from the beginning of the deposition at both deposition temperatures - as expected to larger depths for the higher temperature. As seen further, an enrichment of Co is present in the top-most layers which is the first Si layer at $20^{\circ} \mathrm{C}$ (layer 0 in Figure 2(b)) and the first adsorption layer at $-60^{\circ} \mathrm{C}$ (layer +1 in Figure $2(\mathrm{~d})$ ). Besides, the deposition at RT leads to the development of a subsurface enrichment of Co (about 2-3 Si layers below the surface, see Figure 2(a)). This is similar to results about a subsurface Auenriched phase in a liquid AuSi alloy [27]. In these systems such a configuration is stabilized by the minimization of the free energy, essentially consisting of atomic binding and surface energies, and the entropy of mixing. In solids strain energy enters in addition. At low temperature, a different depth profile is observed. It consists of a diffusion profile, overlaid by oscillations (Figure 2(c)). The oscillations are due to the fact that every second Si layer is Co depleted. This is similar to the model for the diffusion microstructure of $\mathrm{Ni}$ in Si (100) by Chang and Erskine [28] and the distribution of metal atoms in metal alloys like $\mathrm{Cu}_{3} \mathrm{Au}$ [29] close to the surface. A similar behaviour is also observed for the growth of $\mathrm{Fe}$ on $\mathrm{Si}$ (100) at very low coverage at room temperature [30]. Again such a configuration is stabilized by the minimization of the Gibbs-free energy, consisting of atomic binding, strain and surface energies, and the entropy of mixing.

With increasing amounts of deposited Co (see Figures 3 and 4), first various types of partially also stoichiometric silicides appear at the surface until, finally, layers of pure Co grow on top of them. However, great differences are observed as well in the onset of the growth of pure Co layers as in the composition and distribution of the silicides. They seem to depend strongly on the growth conditions, that is, growth at RT, LT, or with Sb as surfactant. While for Co deposition at RT the formation of such a pure layer of Co is first seen at an amount of about $23 \mathrm{ML}$ of deposited Co (Figure 3), it is already observed for amounts of about $6 \mathrm{ML}$ of $\mathrm{Co}$ for LT (Figure 3) and $3 \mathrm{ML}$ of Co for surfactant-mediated deposition (Figure 4). Concerning silicide formation, at RT deposition almost stoichiometric CoSi (below 6ML: $\mathrm{Co}_{0.6} \mathrm{Si}_{0.4}$, above $6 \mathrm{ML}: \mathrm{Co}_{0.5} \mathrm{Si}_{0.5}$ ) forms at the interface which grows thicker and thicker (Figures!3 and 4). Only at high amounts of deposition also other phases form as kind of transition between Si bulk and CoSi, and CoSi and Co at 
Room temperature growth

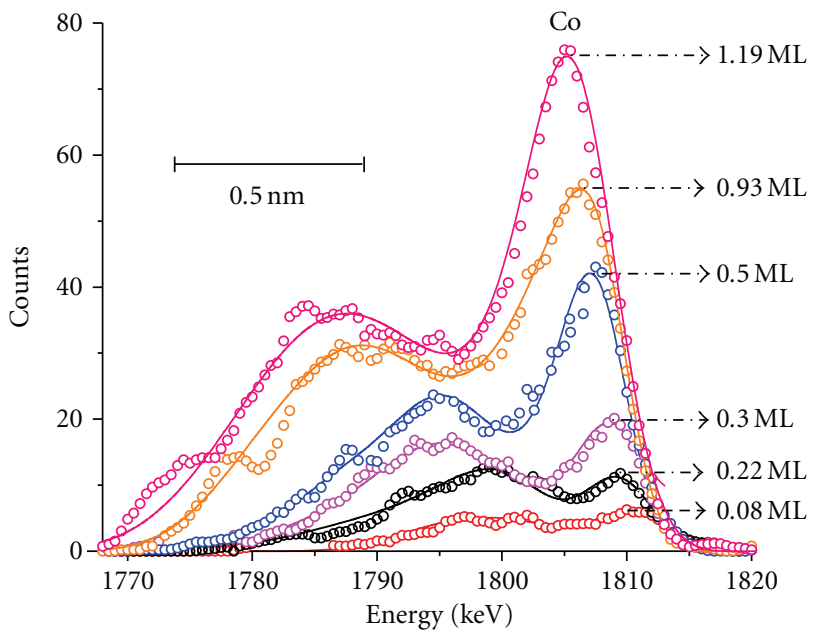

(a)

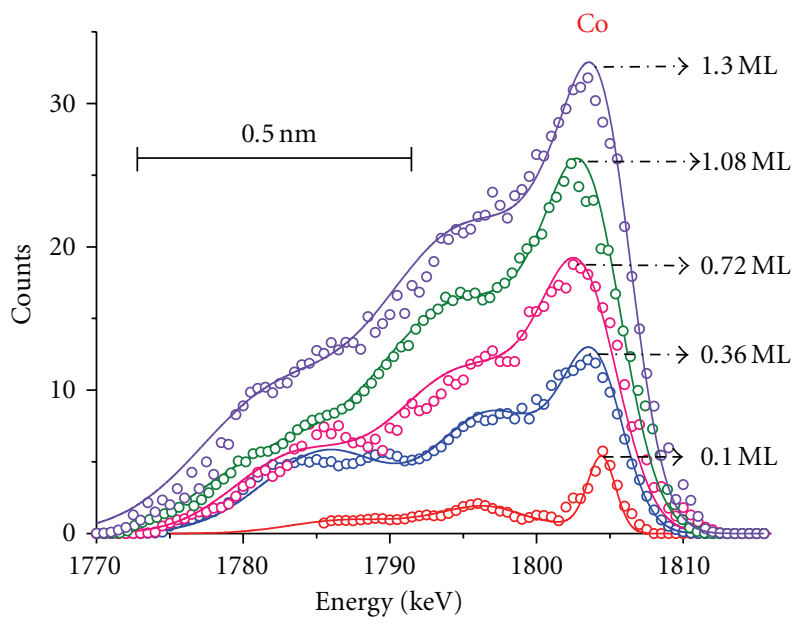

(c)

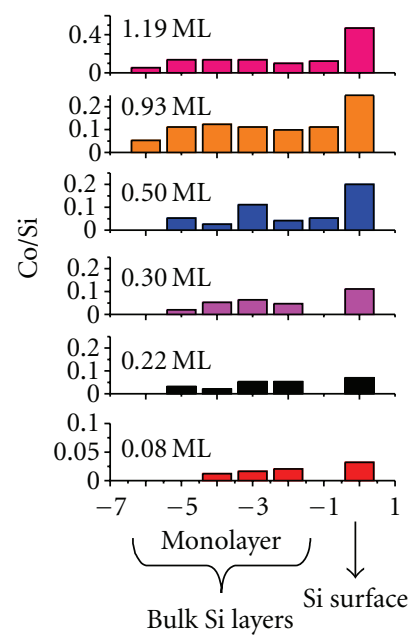

(b)

Low temperature growth

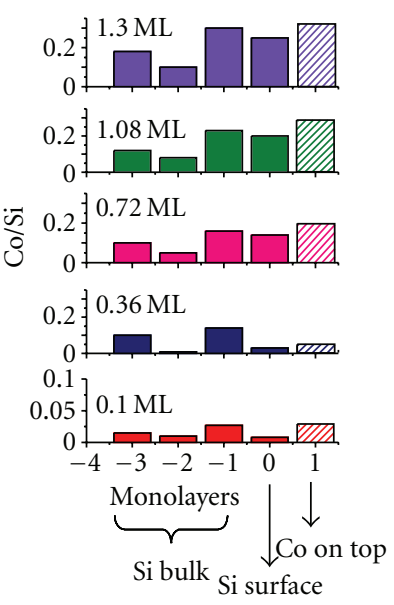

(d)

Figure 2: Co on Si (100), low coverage ( $\leq 1.3 \mathrm{ML})$. Panels at the left-hand side: Co edge of HRBS spectra (circles) and RUMP simulations (solid lines). Panels at the right hand side: Co concentrations as determined by the RUMP simulations versus depth (depth scale: monolayers of Si, each $6.87 \cdot 10^{14}$ at./ $\mathrm{cm}^{2}$ thick). (a) Co deposition at RT. The peaks between 1800 and $1810 \mathrm{keV}$ are due to backscattering from Co at the surface, the other peaks in the range of $1785-1800 \mathrm{keV}$ due to Co atoms in the Si bulk (subsurface enrichment). (b) Co concentration profiles $(\mathrm{Co} / \mathrm{Si}$ ) in subsequent layers of the sample as obtained from (a) by RUMP simulations. Layer 0 is the topmost $\mathrm{Si}$ layer; $-1,-2,-3$, -4 , and -5 are subsequent layers in the bulk. Note that the $y$-axes for 0.08 and $1.19 \mathrm{ML}$ have scales different from others. (c) Co deposition at $-60^{\circ} \mathrm{C}$. The peaks between 1800 and $1805 \mathrm{keV}$ are due to backscattering from Co at the surface. The oscillations in the Co concentration mentioned in the text are clearly visible. (d) Co concentration ( $\mathrm{Co} / \mathrm{Si}$ ) in the various $\mathrm{Si}(100)$ layers of the Si crystal as derived from (c) by RUMP simulations. Layer 1 (hatched column) corresponds to Co atoms on top of the Si crystal, layer 0 is the first Si layer, and layers $-1,-2$, -3 , and -4 are subsequent layers in the Si bulk (solid columns).

the surface: at the Si-CoSi interface a $\mathrm{CoSi}_{2}$-like phase and towards the surface a $\mathrm{Co}_{2} \mathrm{Si}$ phase. This is different for LT and surfactant-mediated deposition. At LT deposition the $\mathrm{Co}_{0.5} \mathrm{Si}_{0.5}$ phase forms at the interface, followed by a lowconcentration tail of Co towards the Si bulk (Figure 3). It is similar to the case of surfactant-mediated deposition. Here the "Co tail" consists of a layer of $10 \mathrm{ML}$ of $\mathrm{CoSi}_{2}$ at the interface and another $7 \mathrm{ML}$ of $\mathrm{CoSi}_{4}$ towards the Si bulk (Figure 4). This interface structure stabilizes at $19 \mathrm{ML}$ of deposited Co and stays like this up to the highest amount of Co deposited in this investigation, that is, $38 \mathrm{ML}$. It is interesting to note that the surfactant $\mathrm{Sb}$ stays, as expected, at 
Low temperature growth

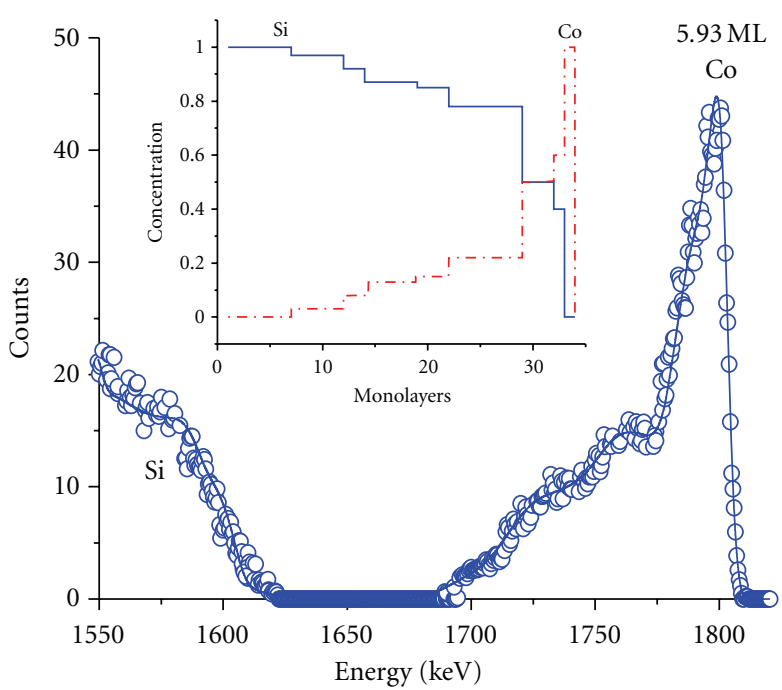

(a)
Room temperature growth

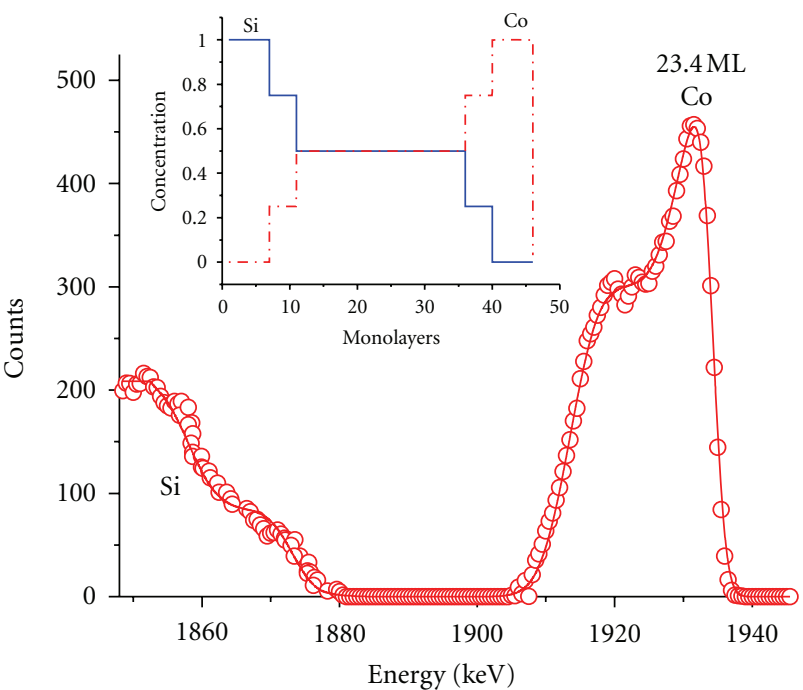

(b)

Figure 3: HRBS spectra of both the Si and Co edges: (a) $5.93 \mathrm{ML}$ of Co deposited at $-60^{\circ} \mathrm{C}$ and (b) $23.42 \mathrm{ML}$ of Co deposited at room temperature $\left(22^{\circ} \mathrm{C}\right)$, together with simulations of the spectra by RUMP (solid lines through the data). The insets show the Si and Co concentrations at the interface as obtained from the RUMP simulations (depth scale: monolayers of Si, each $6.87 \cdot 10^{14}$ at./ $\mathrm{cm}^{2}$ thick). The right hand side of the concentration profiles corresponds to the free surface of the Co/Si. Layer 0 was deliberately put into the Si bulk.

the surface of the sample during deposition (see Figure 4(b)). Only at larger amounts of deposited Co some mixing of $\mathrm{Sb}$ and Co occurs.

In Figure 5, finally, the Co and Si depth profiles for the thickest deposited layers of Co as obtained from the RUMP simulations are compared for the three different deposition conditions. As already stated above, the structures of the CoSi interfaces exhibit marked differences. for Co deposition at RT (23 ML, Figure 5(c)) a broad layer of $\mathrm{Co}_{0.5} \mathrm{Si}_{0.5}(18.8 \AA)$ is formed between two thin transition layers of $\mathrm{CoSi}_{2}(2.2 \AA)$ and $\mathrm{Co}_{2} \mathrm{Si}(3.75 \AA)$. Only a very thin layer of pure Co is present. For Co deposition at LT (5.93 ML, Figure 5(b)) also in this case thin layer of $\mathrm{Co}_{0.5} \mathrm{Si}_{0.5}$ exists at the interface with a thin layer of pure Co on top. (The thinness of this layer is due to the small amount of deposited Co. It would certainly grow for higher amounts of Co.) But here a long tail of different low-Co concentration silicides is observed towards the Si bulk. For surfactant-mediated deposition a similar tail of low-Co concentration silicides is found, but in this case it consists, as said above, of two well-defined silicides with stoichiometric compositions: $10 \mathrm{ML}$ of $\mathrm{CoSi}_{2}$ and $7 \mathrm{ML}$ of $\mathrm{CoSi}_{4}$. The $\mathrm{Co}_{0.5} \mathrm{Si}_{0.5}$ phase, present in the other cases, could not be found.

It should be noted that in all cases flat surfaces are maintained during Co deposition. No roughening is observed; it should show up in rounding off of the high-energy edge of the HRBS spectra [31] which is absent in all cases. At higher amounts of deposited Co even a layer-by-layer growth occurs. This is perhaps most evident from the HRBS spectra of Sb-mediated growth at RT from Figure 4(b). Here the high-energy Si edge shifts almost parallel with increasing Co deposition, since a homogeneous Co film builds up on top. No $\mathrm{Si}$ is found at the surface; only the Co film is getting thicker. This is a clear indication of Sb working as surfactant properly: due to its low surface energy Sb suppresses the segregation of $\mathrm{Si}$ at the surface and apparently also the formation of additional silicides at the interface. This is different for RT deposition of Co without Sb as surfactant. Here right from the beginning a CoSi-like phase is formed continuously, until a large amount of deposited Co (about $16 \mathrm{ML}$ ) first a $\mathrm{Co}_{2} \mathrm{Si}$ phase and finally (after about $23 \mathrm{ML}$ of $\mathrm{Co}$ ) a film of pure Co starts to grow. But also here no $\mathrm{Si}$ is segregating at the surface in the form of a layer of pure Si. This would definitely be visible in the Si HRBS spectrum of Figure 4(a). These observations contrast to a certain degree to the findings of a very recent paper by Pronin et al. [32]. There the authors studied RT deposition of Co on Si (100) by high-resolution photoelectron spectroscopy and a few other techniques in a thorough investigation. The authors claim the segregation of a Si surface layer and, besides, the growth of a layer of pure Co, starting at a Co coverage of about $0.7 \mathrm{~nm}$. Both findings seem not to be in agreement with our observations. It will be interesting to solve this riddle and find a solution which satisfies both investigations.

We finally want to note that there exist differences in the surface energy of the Cobalt spectra presented in different panels. They are due to slightly different incident energies of the ion beam, since the experiments were performed on different days. 
Room temperature growth-no Sb mediated
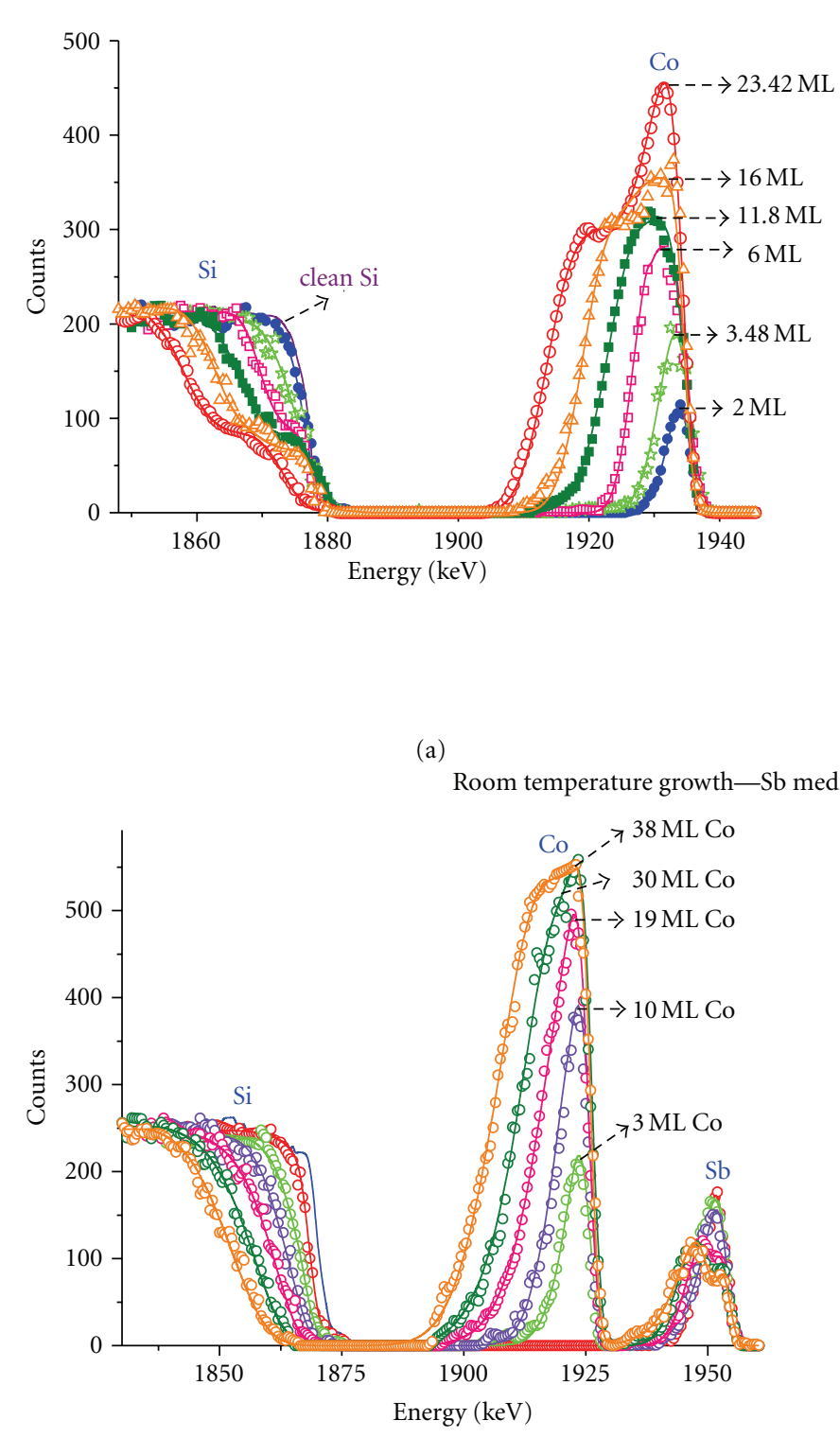

(c)

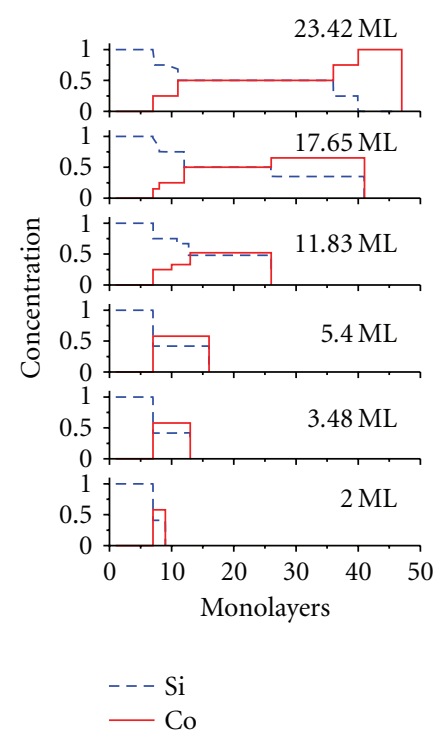

(b)

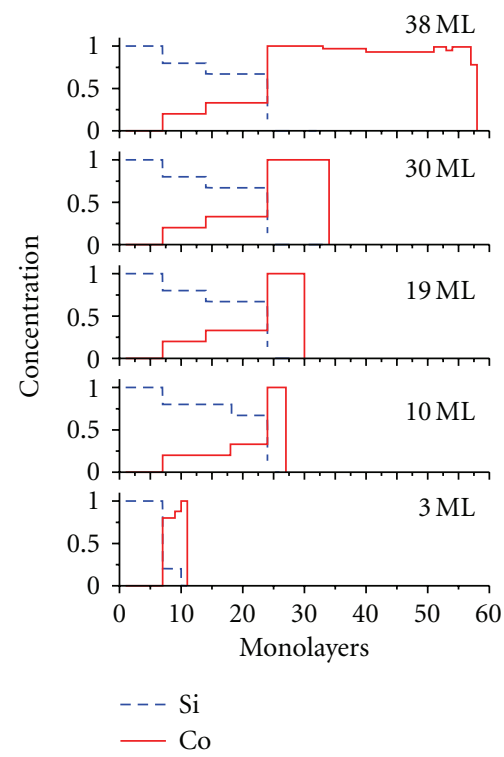

(d)

FIgure 4: Co on Si, higher coverage ( $\geq 2 \mathrm{ML}$ ). Panels at left-hand side: Co and Si (and Sb) HRBS spectra (circles) and RUMP simulations (solid lines). Panels at the right-hand side: Co and Si concentrations as derived by RUMP (depth scale: monolayers of Si, each 6.87 . $10^{14}$ at./ $\mathrm{cm}^{2}$ thick). The right-hand side of the concentration profiles corresponds to the free surface of the samples. (a) Co deposition at RT. (b) Co and Si concentration profiles derived from (a). (c) Surfactant-mediated deposition of Co at RT. (d) Co and Si concentration profiles derived from (c). The right-hand side of the concentration profiles corresponds to the free surface of the samples. The right-hand side of the concentration profiles corresponds to the surface of the Co/Si samples (which in case of the Sb-mediated deposition is below an Sb layer). Layer 0 was deliberately put into the Si bulk.

\section{The Co-Si (100) Interface with a MgO Tunnel Barrier}

3.1. Introduction. As evident, for example, from the results presented in Section 2, Co-Si (100) interfaces are in no way sharp with an abrupt transition between Co and Si but show diffusion of Co and $\mathrm{Si}$ with spontaneous silicide formation at the interface at room temperature $[16,20]$ and even below room temperature [17]. This structural disorder at the interface would drastically reduce the spin-polarization 


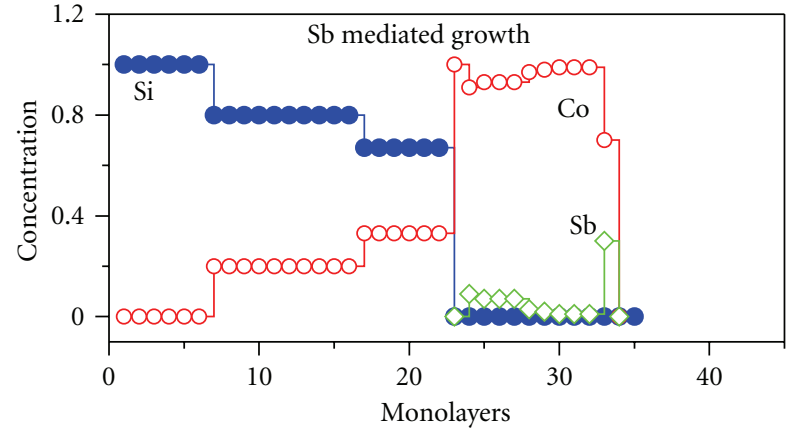

(a)

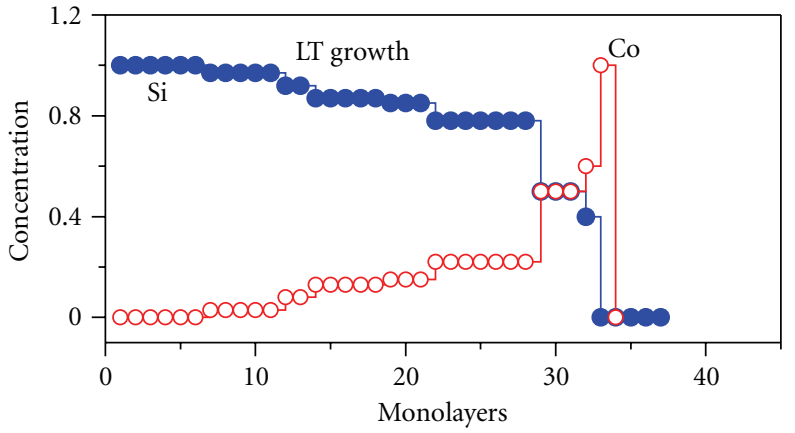

(b)

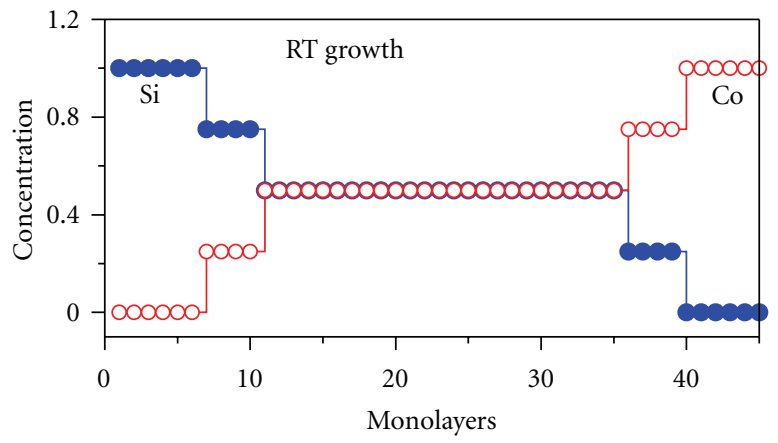

(c)

FIGURE 5: Co and Si (and Sb) concentrations as derived by RUMP simulations from the HRBS data of Figures 3 and 4. (c) Growth of 23.4 ML of Co on Si at room temperature, (b) $5.93 \mathrm{ML}$ of Co deposited at low temperature $\left(-60^{\circ} \mathrm{C}\right)$, and (a) growth of $19 \mathrm{ML}$ of Co on $\mathrm{Si}$ with $\mathrm{Sb}$ as a surfactant. They seem to be representative for the interface structure in a stationary state. The free surface of the three samples is located at the right-most rim of the profiles. Layer 0 was deliberately put into the Si bulk.

at the interface and, hence, the spin injection efficiency [15]. On the other hand, tunnel barriers in between a ferromagnet and a semiconductor have proven to be high-quality spinselective barriers in a prototype GaAs system $[33,34]$ and, most recently, in the case of Si [1,10-12, 35-37]. Using a tunnel barrier on Si has mainly two advantages: (i) it forms a chemical barrier between the FM and the $\mathrm{Si}$ and (ii) it has a good spin-selective tunnel resistance. When an $\mathrm{Al}_{2} \mathrm{O}_{3}$ tunnel contact is used $[1,10-12,35,36]$, the maximum spin injection that can be achieved might be limited by the scattering and depolarization of spin-polarized carriers due to its amorphous nature. An alternative approach for increasing the spin polarization is to use a crystalline $\mathrm{MgO}$ tunnel barrier. There a large spin polarization $(\sim 50 \%)$ was achieved using a $\mathrm{CoFe} / \mathrm{MgO}$ tunnel injector on a GaAs LED structure [34].

In the present study magnesium oxide $(\mathrm{MgO})$ was chosen as tunnel barrier on Si for several reasons. (i) It is chemically inert and thermally stable and should, therefore, result in sharp interfaces with both $\mathrm{Si}$ and ferromagnetic metals, (ii) $\mathrm{MgO}$ has a wide band gap $(7.3 \mathrm{eV})$, ensuring a large band offset with Si to minimize leakage currents, and (iii) the crystalline properties will facilitate coherent tunnelling of spin polarized electrons. $\mathrm{MgO}$ is a highly insulating crystalline solid with $\mathrm{NaCl}$ structure. The lattice constant of $\mathrm{MgO}$ is $4.211 \AA$, whereas that of $\mathrm{Si}$ is $5.431 \AA$, implying a direct lattice mismatch of $-22.5 \%$, but there is a near commensurate match with $\mathrm{Si}$ at a $4: 3$ ratio of the lattice constants (four $\mathrm{MgO}$ to three Si lattice constants). Fork et al. first reported epitaxial growth of thick $\mathrm{MgO}$ films on $\mathrm{Si}$ (100) by pulsed laser deposition [38]. The interface with $\mathrm{Si}$ was found to be incommensurate but abrupt and free from secondary phases or interdiffusion. Also for the case of an ultra-thin $\mathrm{MgO}$ film on $\mathrm{Si}(100)$, the interface is expected to be incommensurate at the early stages of growth due to the presence of large lattice mismatch. So the minimization of the defect density in the epitaxial growth of an ultra-thin $\mathrm{MgO}$ film on $\mathrm{Si}$ (100) can be a real challenge.

For the realization of a high tunnelling magneto-resistive effect, the ultra-thin $\mathrm{MgO}$ barrier on Si should satisfy certain important issues. First, the tunnel barrier should be pinhole free: the requirement for low-resistance tunnel junctions pushes the barrier thickness to lower length scales, making barrier pinholes a real and significant problem. The relative contributions from the two conduction channels-elastic tunneling through the insulating spacer and ballistic spin polarized transport through the narrow pinhole shorts-can also change the magnetoresistive response [39]. Secondly, the thermal stability of the ferromagnetic metals on thin tunnel junctions is important, due to compatibility issues with existing complementary metal-oxide semiconductor CMOS processes, that is, for the production of magnetic random 


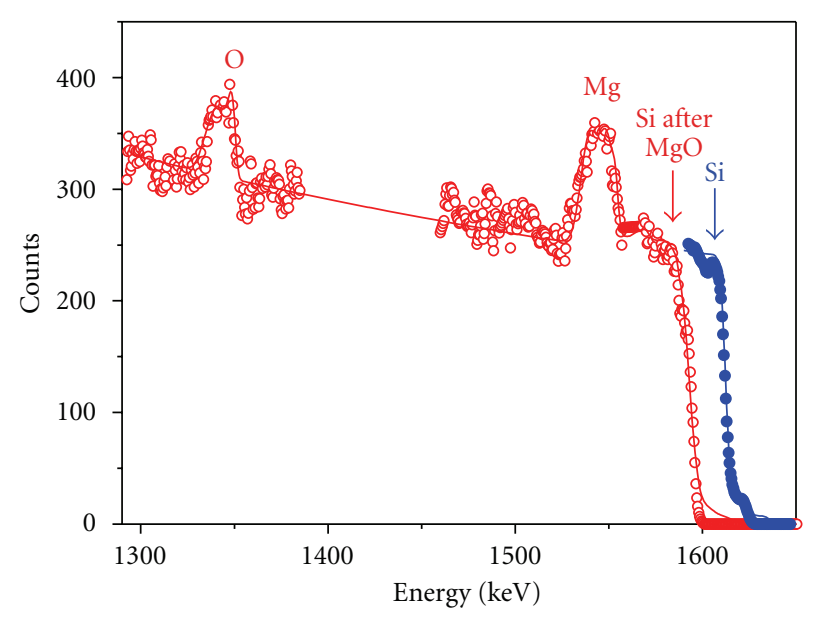

(a)

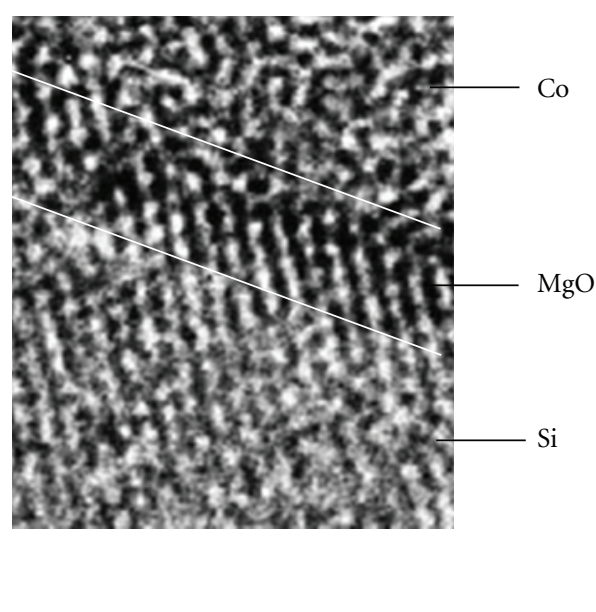

(b)

FIgURE 6: (a) HRBS spectra of a Si sample before and after MgO evaporation. Before evaporation: spectrum of the high-energy Si edge (solid circles) and RUMP simulation (solid line) of the clean Si. After MgO evaporation: spectrum of Si with Mg and O peaks of the $18.3 \mathrm{ML}$ thick MgO film on top (open circles) and RUMP simulation (solid line) of the 18.3 ML thick MgO film as prepared on Si (100) surface. The spectrum shows that the MgO film is very uniform. Surplus oxygen at the surface of the MgO film is clearly visible. (b) HR-TEM crosssectional micrograph of a $\mathrm{Si}(100) / \mathrm{MgO}(18.3 \mathrm{ML}) / \mathrm{Co}$ structure. The lines have been added at the interfaces to aid the eye in distinguishing the layers of small contrast difference.

access memories and for sensor applications where hightemperature operation can be important. Also the thermal stability of magnetic tunnel junctions is of considerable interest because their performance has been shown to be improved by annealing [40]. The third important issue is that of the optimization of the resistance-area product of such tunnel barriers. Fert and Jaffer's calculations reveal that a reasonable value of the magnetoresistance (MR) can only be obtained in the FM/I/Si/I/FM structure if the resistancearea (RA) product of both $\mathrm{FM} / \mathrm{I} / \mathrm{Si}$ contacts is in a relatively narrow range [41].

In Section 3 the growth and characterization of ultra-thin $\mathrm{MgO}$ films are explored on Si (100) by reactive molecular beam epitaxy. Its interface with the Si substrate and the ferromagnetic metal has been studied by in situ HRBS and ex situ HR-TEM. The thermal stability of ultra-thin Co (and also $\mathrm{Fe}$ ) films on such $\mathrm{MgO}$ tunnel barriers has been verified by thermal annealing and in situ HRBS measurements. The issue of tailoring the resistance-area product of the tunnel contact has been addressed by down-scaling the barrier thickness to the sub-nanometer regime and, in other way, by producing oxygen deficient tunnel barriers.

3.2. Experimental. Equilibrium thermodynamic data suggest that $\mathrm{MgO}$ is stable against the formation of interfacial compounds with Si. But in the case of reactive molecular beam epitaxy where metallic $\mathrm{Mg}$ is used as the cation source along with (an oxidising background source of) $\mathrm{O}_{2}$ gas, kinetic limitations may supplant equilibrium considerations. Potential aggravating effects are (i) substrate oxidation prior to initiation of film growth and (ii) magnesium silicide formation at the interface. These effects correspond to the two extremes in growth conditions, requiring a better understanding of their relative importance. It is therefore important to find an appropriate growth regime that at least partially overcomes these effects. In the sample preparation, precautions were taken to avoid the above mentioned effects by a particular growth procedure. First $0.5 \mathrm{ML}$ of $\mathrm{Mg}$ was evaporated on cleaned Si (100) which is expected to occupy the cave sites (see Figure 1) on the $\mathrm{Si}$ (100) surface without any silicide formation [42-44] and then oxygen was streamed into the chamber. In this way both, silicide formation and also the oxidation of $\mathrm{Si}$ could be avoided. The growth of the stoichiometric $\mathrm{MgO}$ films was then continued by evaporating $\mathrm{Mg}$ at a rate of $1 \mathrm{ML} / \mathrm{min}$ at an oxygen pressure of $1 \times 10^{-7} \mathrm{mbar}$. On top of these $\mathrm{MgO}$ films thin films of $\mathrm{Co}(3 \mathrm{ML})$ or Fe (4 ML) were deposited by evaporation with a Knudsen cell in a some cases.

For characterizing these ultra-thin $\mathrm{MgO}$ tunnel barriers in atomic detail again in situ HRBS was used. The measurements were carried out in the same way as described in Section 2.2. $2 \mathrm{MeV} \mathrm{N}^{+}$ions at an incidence angle of $10^{\circ}$ to the sample surface and a scattering angle of $37.5^{\circ}$ were used for the analysis, if not stated otherwise. n-type Si (100) samples with resistivity 4-10 $\Omega-\mathrm{cm}$ (P doped) were cleaned in UHV by flash heating at $1050^{\circ} \mathrm{C}$. The surface cleanness of the $\mathrm{Si}$ samples was verified by HRBS measurements. Before use the effusion cell was outgassed; no $\mathrm{C}$ or $\mathrm{O}$ contaminants were found during evaporation. The evaporation rates of the metals to be evaporated, $\mathrm{Mg}$, $\mathrm{Co}$, and $\mathrm{Fe}$, were calibrated by HRBS with an accuracy of about 5\%. In addition HR-TEM measurements were carried out to reveal the structure of the $\mathrm{MgO}$ tunnel barrier and its interfaces.

\subsection{Results and Discussion}

3.3.1. MgO Tunnel Barrier on Si. Figure 6(a) shows the HRBS spectra of the Si sample before and after the evaporation 


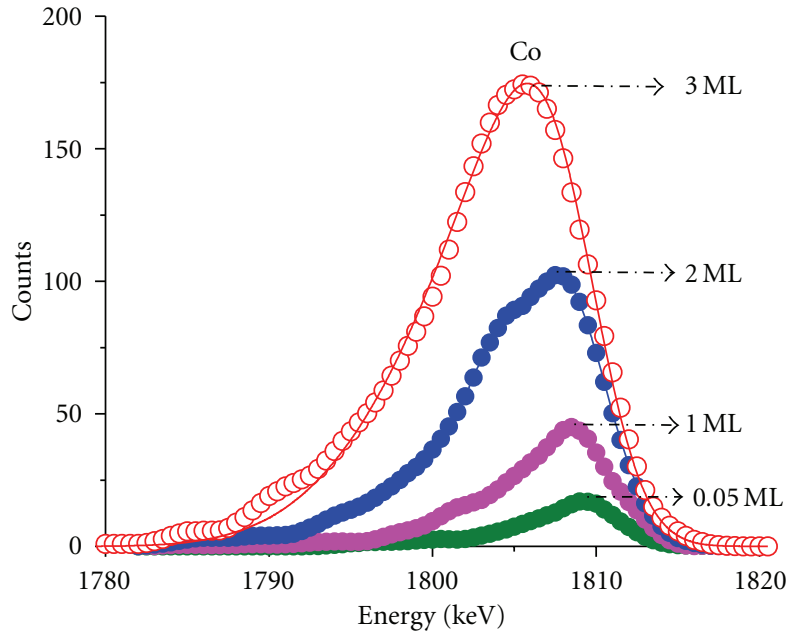

(a)

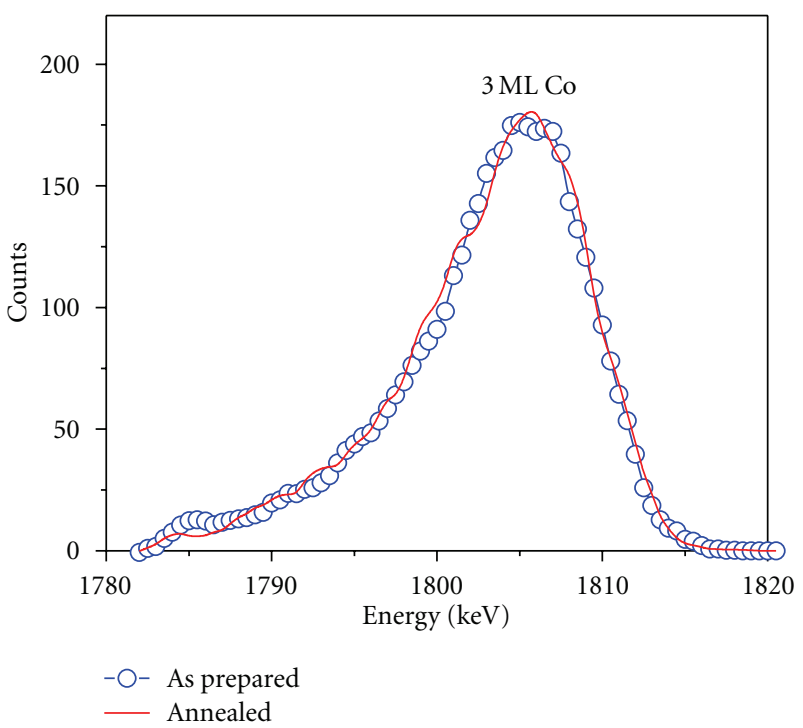

(c)

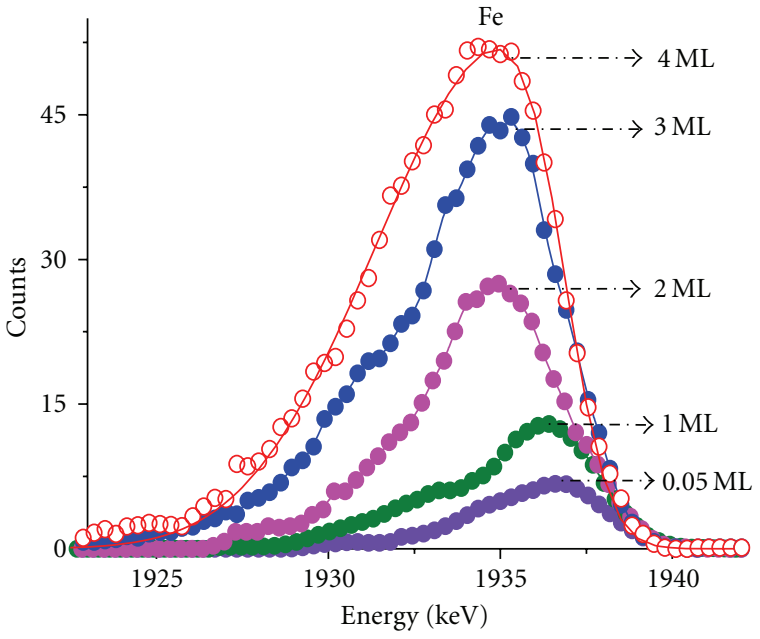

(b)

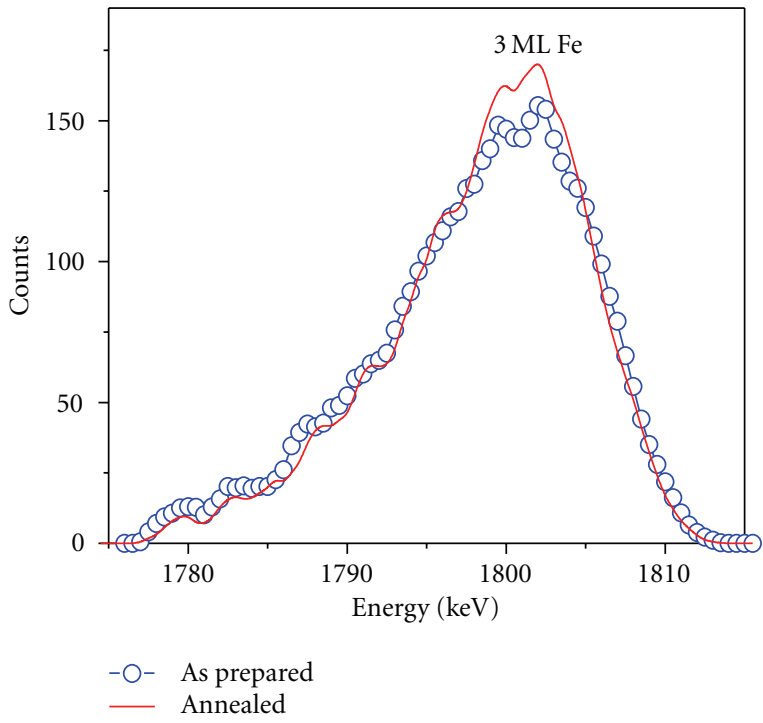

(d)

FIGURE 7: HRBS spectra of grown and thermal stability of ferromagnetic metal films (Co, Fe) on a MgO tunnel barrier/Si (100) structure. (a) Growth of $\mathrm{Co}(0.05-3 \mathrm{ML})$ using $2 \mathrm{Mev} \mathrm{N}^{+}$ions for analysis at an incidence angle of $8^{\circ}$. RUMP simulation is also shown for $3 \mathrm{ML}$ of Co. (b) Growth of $\mathrm{Fe}(0.05-4 \mathrm{ML})$ using $2 \mathrm{Mev} \mathrm{He}^{+}$ions at an incidence angle of $3^{\circ}$. RUMP simulation is also shown for $4 \mathrm{ML}$ of Fe. (c) $\mathrm{HRBS}$ spectra of $3 \mathrm{ML}$ of Co as prepared (open circles) and after annealing at $450^{\circ} \mathrm{C}$ for $15 \mathrm{~min}$ (solid line). (d) HRBS spectra of $3 \mathrm{ML}$ of Fe as prepared (open circles) and after annealing at $450^{\circ} \mathrm{C}$ for $15 \mathrm{~min}$ (solid line). No major changes are visible.

of $18 \mathrm{ML}$ of $\mathrm{MgO}$. The high-energy edge of the clean $\mathrm{Si}$ sample is at $1620 \mathrm{kev}$. After the evaporation of the $\mathrm{MgO}$ layer the Si surface edge is shifted almost parallel to lower energies $(1600 \mathrm{keV})$, besides a slight kink at about $2 / 3$ of the spectrum height (see the following). The two peaks, observed on the Si background spectrum at $1560 \mathrm{keV}$ and $1350 \mathrm{keV}$, are due to the $\mathrm{Mg}$, and $\mathrm{O}$ in the grown $\mathrm{MgO}$ thin film on the Si substrate, respectively. The spectrum was simulated by the program RUMP [26], in order to obtain more detailed information about the thickness and composition of the $\mathrm{MgO}$ film, and its interface structure with Si. In these simulations the sample was subdivided into thin sublayers of the thickness of $1 \times 10^{15}$ atoms $/ \mathrm{cm}^{2}$. The composition of each sublayer was varied and the HRBS spectrum calculated for the assumed $\mathrm{Mg}$ and $\mathrm{O}$ concentrations and depth distributions until good agreement with the experimental data was achieved. According to these results the grown $\mathrm{MgO}$ film on the average is about 18.3 ML thick, has a more or less sharp interface with $\mathrm{Si}$ (uncertainty about $1 \mathrm{ML}$ ), and exhibits the composition $\mathrm{Mg}_{0.5} \mathrm{O}_{0.5}$, with the exception of a very thin surface layer $(2 \mathrm{ML})$ which has the composition $\mathrm{Mg}_{0.3} \mathrm{O}_{0.7}$, that is, exhibits an $\mathrm{O}$ surplus (roughly 1 complete $\mathrm{ML}$ ). This increased $\mathrm{O}$ content of the surface layer is well visible in the oxygen spectrum of Figure 6(a) and may be due to the fact that some residual oxygen gas was left in the preparation chamber after closing the oxygen bottle and so on the surface of the $\mathrm{MgO}$ film.

The almost parallel shift of the high-energy edge of Si gives strong evidence that the $\mathrm{MgO}$ layer is quite uniform in thickness and that no excessive island formation has 
occurred. From the RUMP simulation, a thickness fluctuation of the $\mathrm{MgO}$ thin film of only $2 \mathrm{ML}$ is found (indeed the slight kink in the Si slope mentioned above indicates that about $1 / 3$ of the $\mathrm{MgO}$ layer has a thickness of $19 \mathrm{ML}$ and $2 / 3$ a thickness of $18 \mathrm{ML})$. It is to be noted that in case of the presence of excessive island growth the Si high-energy edge would not shift in such a parallel manner. Besides, the almost trapezoidal shapes of the $\mathrm{Mg}$ and $\mathrm{O}$ parts of the spectrum are indicative of a very homogeneous $\mathrm{MgO}$ layer; here, island growth would have resulted in a more triangular shape. From the comparison with the RUMP simulation the $\mathrm{MgO}-\mathrm{Si}$ interface was found to be very sharp, hence ruling out the formation of extended $\mathrm{Mg}$-silicide phases or a strong oxidation of the Si surface.

In general, the presence of pin-holes in ultra-thin films is a real and serious problem [39]. In order to further verify the very positive properties of the MgO barrier, HR-TEM studies were carried out. Figure 6(b) shows an HR-TEM micrograph of a $\mathrm{Co} / \mathrm{MgO}(18 \mathrm{ML}) / \mathrm{Si}(100)$ heterostructure. The image shows a good morphology with rather smooth and flat layers. the $\mathrm{MgO}$ layer has crystalline structure and a very sharp interface with $\mathrm{Si}$. The results confirm the good homogeneity of the film derived from the HRBS results. TEM pictures taken from various parts of the film (not shown here) show that this $\mathrm{MgO}$ film does not have pin-holes.

3.3.2. Growth of Ferromagnetic Metal on MgO Tunnel Barrier. Thin layers of the ferromagnetic metals $\mathrm{Co}$ and $\mathrm{Fe}$ were grown on these $\mathrm{MgO}$ tunnel barriers. The layers were characterized in situ by $\mathrm{HRBS} 2 \mathrm{MeV} \mathrm{N}^{+}$ions at an incidence angle of $8^{\circ}$ and $2 \mathrm{MeV} \mathrm{He}^{+}$ions at an incidence angle of $3^{\circ}$ to the sample surface for Co and Fe cases, respectively. The scattering angle was $37.5^{\circ}$ for both cases. The HRBS spectra at the energy edges of $\mathrm{Co}$ and $\mathrm{Fe}$ are shown in Figures 7(a) and 7(b), respectively, for various film thicknesses. From the very beginning of growth of both Co and Fe (0.05 ML), island growth is evident because of the presence of long tails of the HRBS peaks towards lower energies. This is quite understandable because of very different surface-free energies of $\mathrm{MgO}\left(1.1 \mathrm{~J} / \mathrm{m}^{2}\right)$ at the one hand and $\mathrm{Co}$ or $\mathrm{Fe}$ $\left(\sim 2.9 \mathrm{~J} / \mathrm{m}^{2}\right)$ on the other hand. So layer-by-layer growth is not favoured for ferromagnetic metals on $\mathrm{MgO}$. In order to obtain detailed information about the growth, the HRBS spectra of the $3 \mathrm{ML} \mathrm{Co}$ and $4 \mathrm{ML}$ Fe thin films were simulated by the program RUMP with the built-in routine "FUZZ" which simulates thickness fluctuations in a layer. The data could best be fitted by assuming the growth of pure ferromagnetic metal $(\mathrm{Co}, \mathrm{Fe})$ films of a roughness of $\sim 2 \mathrm{ML}$ (standard deviation).

\subsubsection{Thermal Stability of Ferromagnetic Films on the $\mathrm{MgO}$} Tunnel Barriers. The thermal stability of the MBE-grown ferromagnet-MgO tunnel junctions on $\mathrm{Si}$ (100) was studied by annealing them at $450^{\circ} \mathrm{C}$ for $15 \mathrm{~min}$ and analyzing them in situ by HRBS. The HRBS spectra of the $3 \mathrm{ML}$ Co and $4 \mathrm{ML}$ Fe films evaporated on such $\mathrm{MgO}$ tunnel barriers are shown in Figures 7(c) and 7(d) in the as-prepared and the annealed state. As evident from the figures, the Co and Fe profiles

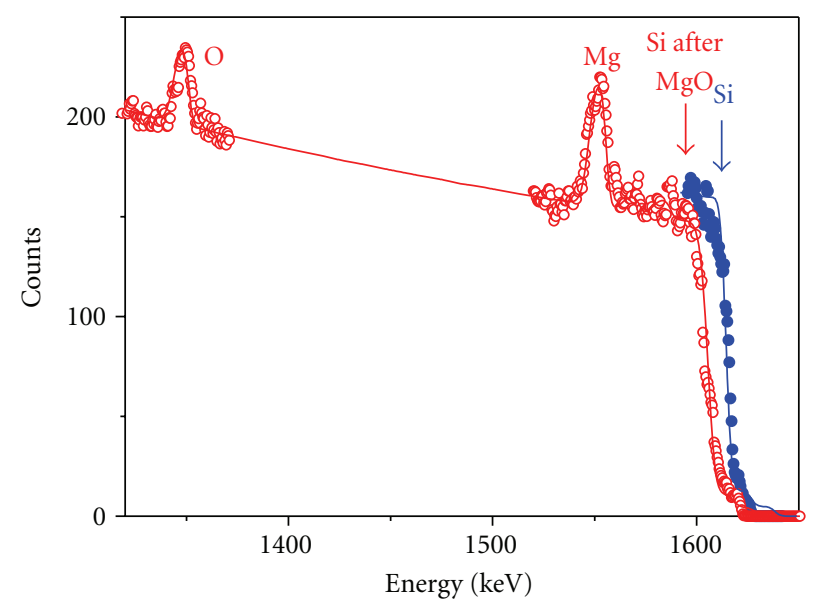

FIgURE 8: Sub-nanometer thick MgO: HRBS spectra before and after $\mathrm{MgO}$ evaporation. HRBS spectra at the Si edge before $\mathrm{MgO}$ evaporation (solid circles) and RUMP simulation (solid line) of the clean Si. After MgO evaporation: HRBS spectra at the $\mathrm{Si}, \mathrm{Mg}$, and O edges (open circles) and RUMP simulation (solid line) of the 7.7 ML thin $\mathrm{MgO}$ layer as prepared on the $\mathrm{Si}(100)$ surface.

do not show any change upon annealing. This means that they are thermally stable up to $450^{\circ} \mathrm{C}$. The slight increase in the maximum count rate of the Fe spectrum after annealing is probably due to the fact that the Fe film becomes more homogeneous after annealing. The great thermal stability of these Co and Fe films should be compared with the thermal behaviour of Co and Fe films on pure Si (100) substrates. In the latter case strong in-diffusion of the metals and outdiffusion of $\mathrm{Si}$ is observed which leads to pronounced silicide formation (see Section 2 and [44, 45]). Nothing like this is observed in the present case which means that the barrier is stable against diffusion and does not show pin holes to a larger amount.

3.3.4. Optimization of Resistance-Area Product. The issue of tailoring the resistance-area product of a ferromagnetic metal-MgO-Si structure has been addressed by (i) scaling down the thickness of the $\mathrm{MgO}$ film into the sub-nanometer regime and (ii) producing tunnel barriers having less oxygen content. Both procedures should result in a reduced resistivity of the tunnel barrier.

(i) Downscaling of MgO Tunnel Barrier Thickness. Following the motivation to decrease the resistance-area product of the tunnel junctions, an ultra-thin $\mathrm{MgO}$ tunnel barrier with a thickness in the sub-nanometer regime has been fabricated. As the oxide thickness is decreased below $1 \mathrm{~nm}$, meeting the reliability specifications becomes even more challenging.

The HRBS spectrum of an ultra-thin and stoichiometric $\mathrm{MgO}$ tunnel barrier prepared on $\mathrm{Si}$ (100) is shown in Figure 8, before and after the evaporation of $\mathrm{MgO}$. After evaporation of $\mathrm{MgO}$, the Si surface edge is shifted almost parallel towards lower energies, besides again a slight kink at about $1 / 3$ of the height of the Si spectrum. This kink indicates 


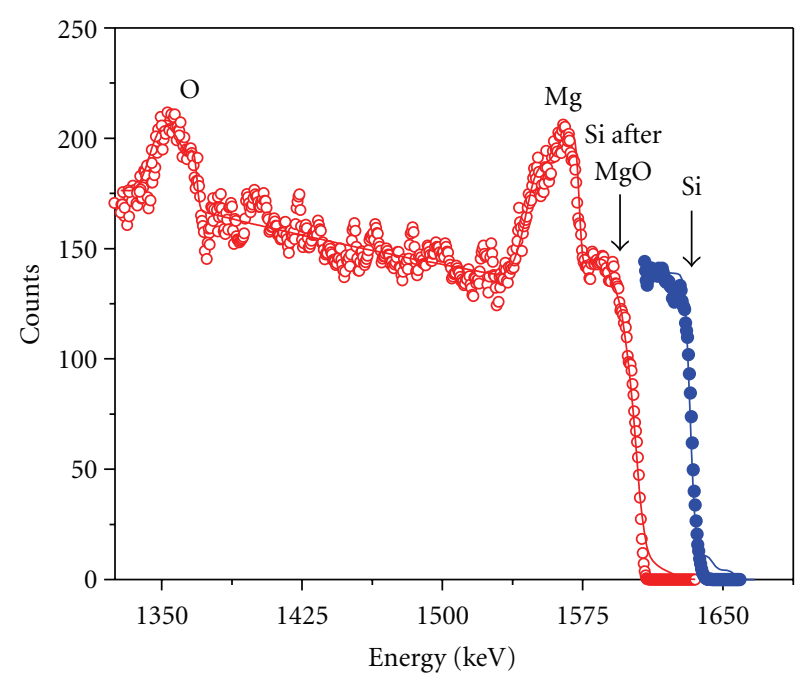

(a)

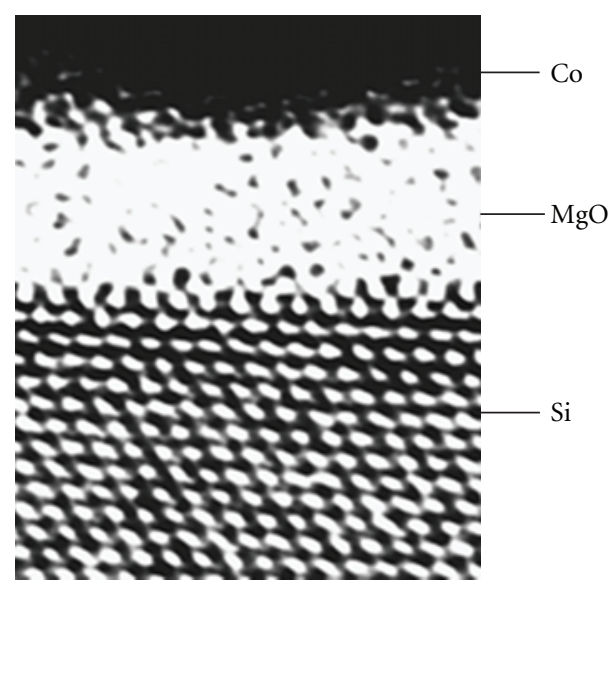

(b)

FIGURE 9: (a) HRBS spectra of a Si sample before and after MgO evaporation. Before evaporation: HRBS spectrum of the high-energy edge Si edge (solid circles) and RUMP simulation (solid line) of the clean Si. After MgO evaporation: HRBS spectrum at Si, Mg and O edges (open circles) and RUMP simulation (solid line) of the $23 \mathrm{ML} \mathrm{MgO}$ as prepared on a Si (100) surface. (b) HR-TEM cross-sectional micrograph of a Si (100)/MgO (23 ML)/Co structure.

that about $2 / 3$ of the film has a thickness of $8 \mathrm{ML}$ and about $1 / 3$ a thickness of $7 \mathrm{ML}$. The two peaks at $1560 \mathrm{keV}$ and $1350 \mathrm{keV}$ are again due to $\mathrm{Mg}$ and $\mathrm{O}$ in the $\mathrm{MgO}$ thin film, respectively. The grown $\mathrm{MgO}$ film is on the average about 7.7 ML thick with a thickness fluctuation of about $1 \mathrm{ML}$, has a rather sharp interface with $\mathrm{Si}$, and exhibits a composition of $\mathrm{Mg}_{0.5} \mathrm{O}_{0.5}$. The (almost parallel) shift of the Si surface edge towards lower energy is due to complete coverage of the $\mathrm{Si}$ surface by the $\mathrm{MgO}$ layer. The MgO-Si interface was found to be rather sharp from the simulation (uncertainty about $1 \mathrm{ML}$ ), hence ruling out the formation of an extended silicide phase or strong oxidation of the Si surface.

(ii) Oxygen Deficient Tunnel Barrier. In another step to tailor the resistance of the $\mathrm{MgO}$ tunnel barrier, an oxygen deficient tunnel barrier was prepared. In the preparation by reactive molecular beam epitaxy, first a few monolayers of stoichiometric $\mathrm{MgO}$ were grown and then the $\mathrm{O}$ content was decreased towards the surface. Such an oxygen-deficient tunnel barrier has two advantages: (1) it will exhibit a lower resistance; (2) it will not oxidize the ferromagnetic metal evaporated on it. The oxygen-deficient $\mathrm{MgO}$ barrier was again characterized in situ by HRBS and ex situ by HRTEM to get in-depth information about the composition, thickness, interface quality, and structure.

Figure 9(a) shows the HRBS spectra before and after the evaporation of $\mathrm{MgO}$ onto the cleaned Si (100) surface. After evaporation of $\mathrm{MgO}$ the Si surface edge is again shifted almost parallel towards lower energies. There are some slight deviations from parallel that cannot be resolved too well and seem to indicate some thickness fluctuations of the film (of the order of 1-2 ML). The two peaks observed on the Si background, at $1560 \mathrm{keV}$ and $1350 \mathrm{keV}$, which are due to $\mathrm{Mg}$ and $\mathrm{O}$ in the grown $\mathrm{MgO}$ thin film, now show a different structure than in case of Figure 6(a). According to the RUMP simulation the grown $\mathrm{MgO}$ film is on the average $23 \mathrm{ML}$ thick. The composition of $\mathrm{MgO}$ is found to be stoichiometric $\mathrm{Mg}_{0.5} \mathrm{O}_{0.5}$ at the interface, but the surface is deficient of oxygen and enriched with $\mathrm{Mg}$ as desired. Besides, this can be directly seen from the spectra. Further, the almost parallel shift of the high-energy edge of Si gives strong evidence that the $23 \mathrm{ML} \mathrm{MgO}_{x}$ layer is quite uniform in thickness (as stated above with a thickness fluctuation of 1-2 ML) and no extensive island formation has occurred. The $\mathrm{MgO}-\mathrm{Si}$ interface is found to be rather sharp from the RUMP simulation, hence ruling out the formation of any extended silicide phase or strong oxidation of the Si surface.

Figure 9(b) shows the high-resolution TEM micrograph of the $\mathrm{Co} / \mathrm{MgO}_{x} / \mathrm{Si}(100)$ heterostructure. The image shows a good morphology with extremely smooth, flat layers and free of pin-holes. This result is in quite good agreement with the HRBS analysis. But the MgO film in this case is found to be amorphous in structure. So decreasing the oxygen content in the grown $\mathrm{MgO}$ tunnel barrier apparently ended with an amorphous structure.

\section{Summary}

In the first part of this paper results about the preparation and investigation of Co-Si (100) Schottky interfaces are presented. The Schottky contacts were prepared in three ways: by evaporation at room temperature (RT), at $-60^{\circ} \mathrm{C}$ (LT), and at RT with Sb as surfactant. Their structures were in situ studied by high-resolution RBS (HRBS). In all cases Co diffuses into the Si substrate right from the beginning, but to a lesser degree at LT and with $\mathrm{Sb}$ as surfactant. For small amounts of deposited Co (less than about 1.3 ML) a subsurface enrichment of Co is observed for RT deposition, 
but for LT deposition kind of a diffusion profile with overlaid oscillations. These oscillations are due to the suppression of Co in every second Si layer.

At higher amounts of deposited Co, the formation of various types of silicides is observed. But there exist big differences, depending on the deposition technique. For RT deposition about stoichiometric CoSi is formed at the interface. Its thickness continuously grows with the deposition until finally (at around $24 \mathrm{ML}$ of Co) metallic Co is formed at the surface. Only very thin layers of other silicides grow on both sides of the CoSi layer. For LT deposition already at about $6 \mathrm{ML}$ of deposited Co metallic Co grows at the surface. It is separated from the Si bulk by a thin layer of CoSi and a subsequent tail of various low-Co content silicides. For Sbmediated deposition already at $3 \mathrm{ML}$ of deposited Co metallic Co grows at the surface. Also in this case a long tail consisting of various low-Co content silicides extends towards the $\mathrm{Si}$ bulk, but no stoichiometric CoSi is formed. As expected, the $\mathrm{Sb}$ surfactant floats at the Co surface most of the time. Only for large amounts of deposited Co some mixing occurs.

In the second part the fabrication and characterization of ultra-thin crystalline MgO tunnel barriers on $\mathrm{Si}$ (100) and some of the important properties required for tunnel barriers on $\mathrm{Si}$ have been addressed. Ultra-thin stoichiometric $\mathrm{MgO}$ tunnel barriers prepared on Si (100) by reactive molecular beam epitaxy are found to be very homogeneous, without pin-holes, crystalline in structure, and to have a sharp interface with the Si (100) substrate. Co and Fe on such a $\mathrm{MgO}$ tunnel barrier were found to have island-like growth with a rough surface, but to be quite stable up to $450^{\circ} \mathrm{C}$, which is important for the integration into integrated circuits. In a move to decrease the resistance of the tunnel barriers, we have fabricated sub-nanometer thin tunnel barriers and oxygen-deficient tunnel barriers. Ultra-thin tunnel barriers of sub-nanometer thickness could be prepared with a sharp interface to the Si substrate with $\sim 2 \mathrm{ML}$ roughness. Oxygendeficient tunnel barriers were found to be amorphous, but also with a sharp interface with $\mathrm{Si}$ (100) and quite homogeneous. These ferromagnet/MgO/Si (100) systems are promising for spin injection into $\mathrm{Si}$.

\section{Acknowledgments}

The authors would like to acknowledge M. Bechtel, Dr. F. Philipp, Dr. Á. Szőkefalvi-Nagy of the MPI IS (former MPI $\mathrm{MF}$ ), and Alaka Tripathy for kind cooperation.

\section{References}

[1] S. P. Dash, S. Sharma, R. S. Patel, M. P. de Jong, and R. Jansen, "Electrical creation of spin polarization in silicon at room temperature," Nature, vol. 462, no. 7272, pp. 491-494, 2009.

[2] S. Datta and B. Das, "Electronic analog of the electro-optic modulator," Applied Physics Letters, vol. 56, no. 7, pp. 665-667, 1990.

[3] S. A. Wolf, D. D. Awschalom, R. A. Buhrman et al., "Spintronics: a spin-based electronics vision for the future," Science, vol. 294, no. 5546, pp. 1488-1495, 2001.
[4] C. Chappert, A. Fert, and F. N. van Dau, "The emergence of spin electronics in data storage," Nature Materials, vol. 6, no. 11, pp. 813-823, 2007.

[5] J. Fabian, A. Matos-Abiague, C. Ertler, P. Stano, and I. Žutić, "Semiconductor spintronics," Acta Physica Slovaca, vol. 57, no. 4-5, pp. 565-907, 2007.

[6] I. Žutić, J. Fabian, and S. D. Sarma, "Spintronics: fundamentals and applications," Reviews of Modern Physics, vol. 76, no. 2, pp. 323-410, 2004.

[7] R. Jansen, B. C. Min, and S. P. Dash, "Oscillatory spinpolarized tunnelling from silicon quantum wells controlled by electric field," Nature Materials, vol. 9, no. 2, pp. 133-138, 2010.

[8] R. J. Soulen, J. M. Byers, M. S. Osofsky et al., "Measuring the spin polarization of a metal with a superconducting point contact," Science, vol. 282, no. 5386, pp. 85-88, 1998.

[9] A. G. Arnov and G. E. Pikus, "Spin injection into semiconductors," Soviet Physics-Semiconductors, vol. 10, no. 6, pp. 698700, 1976.

[10] S. P. Dash, S. Sharma, J. C. Le Breton et al., "Spin precession and inverted Hanle effect in a semiconductor near a finiteroughness ferromagnetic interface," Physical Review B, vol. 84, no. 5, Article ID 054410, 11 pages, 2011.

[11] R. Jansen, B. C. Min, S. P. Dash et al., "Electrical spin injection into moderately doped silicon enabled by tailored interfaces," Physical Review B, vol. 82, no. 24, Article ID 241305, 4 pages, 2010.

[12] S. P. Dash, S. Sharma, J. C. Le Breton, and R. Jansen, "Silicon spintronics at room temperature," in Spintronics III, vol. 7760 of Proceedings of SPIE, San Diego, Calif, USA, August 2010.

[13] R. S. Patel, S. P. Dash, M. P. de Jong, and R. Jansen, "Magnetic tunnel contacts to silicon with low-work-function ytterbium nanolayers," Journal of Applied Physics, vol. 106, no. 1, Article ID 016107, 2009.

[14] I. Žutić and J. Fabian, "Spintronics: silicon twists," Nature, vol. 447, no. 7142, pp. 269-270, 2007.

[15] G. Kioseoglou, A. T. Hanbicki, R. Goswami et al., "Electrical spin injection into Si: a comparison between Fe/Si Schottky and $\mathrm{Fe} / \mathrm{Al}_{2} \mathrm{O}_{3}$ tunnel contacts," Applied Physics Letters, vol. 94, no. 12, Article ID 122106, 2009.

[16] S. P. Dash, D. Goll, and H. D. Carstanjen, "Subsurface enrichment of Co in Si (100) at initial stages of growth at room temperature: a study by high-resolution Rutherford backscattering," Applied Physics Letters, vol. 90, no. 13, Article ID 132109, 2007.

[17] S. P. Dash, D. Goll, and H. D. Carstanjen, "Near-surface compositional oscillations of Co diffused into $\mathrm{Si}(100)$ at 60 oc: a study by high-resolution Rutherford backscattering," Applied Physics A, vol. 91, no. 3, pp. 379-383, 2008.

[18] S. P. Dash, D. Goll, and H. D. Carstanjen, "Improvement of interface structure and magnetic properties of Co on $\mathrm{Si}(100)$ by surfactant (Sb) mediated growth," Applied Physics A, vol. 97, no. 3, pp. 651-656, 2009.

[19] W. S. Cho, J. Y. Kim, N. G. Park et al., "Atomic structure of ultrathin Co layer on $\mathrm{Si}(001)(2 \times 1)$ at room temperature," Surface Science, vol. 453, no. 1-3, pp. L309-L314, 2000.

[20] H. L. Meyerheim, U. Döbler, A. Puschmann, and K. Baberschke, "Preparation-dependent $\mathrm{Co} / \mathrm{Si}(100)(2 \times 1)$ interface growth: spontaneous silicide formation versus interstitial-site mechanism," Physical Review B, vol. 44, p. 5738, 1990.

[21] G. Rangelov, P. Augustin, J. Stober, and T. Fauster, "Initial stages of epitaxial $\mathrm{CoSi}_{2}$ formation on $\mathrm{Si}(100)$ surfaces," Physical Review B, vol. 49, no. 11, pp. 7535-7542, 1994. 
[22] V. Scheuch, B. Voigtländer, and H. P. Bonzel, "Nucleation and growth of $\mathrm{CoSi}_{2}$ on $\mathrm{Si}(100)$ studied by scanning tunneling microscopy," Surface Science, vol. 372, no. 1-3, pp. 71-82, 1997.

[23] M. V. Gomoyunova, I. I. Pronin, N. R. Gall, S. L. Molodtsov, and D. V. Vyalykh, "Silicon surface reconstruction lost upon cobalt adsorption,” Technical Physics Letters, vol. 29, no. 6, pp. 496-499, 2001.

[24] A. P. Horsfield, S. D. Kenny, and H. Fujitani, "Densityfunctional study of adsorption of Co on Si(100)," Physical Review B, vol. 64, no. 24, Article ID 245332, pp. 24533212453326, 2001.

[25] T. Enders, M. Rilli, and H. D. Carstanjen, "A high-resolution electrostatic spectrometer for the investigation of near-surface layers in solids by high-resolution Rutherford backscattering with $\mathrm{MeV}$ ions," Nuclear Instruments and Methods in Physics Research B, vol. 136, p. 1183, 1992.

[26] L. R. Doolittle and M. O. Thompson, RUMP, Computer Graphics Service, 2002.

[27] O. G. Shpyrko, R. Streitel, V. S. K. Balagurusamy et al., "Surface crystallization in a liquid AuSi alloy," Science, vol. 313, no. 5783, pp. 77-80, 2006.

[28] Y. J. Chang and J. L. Erskine, "Diffusion-layer microstructure of Ni on Si(100)," Physical Review B, vol. 26, no. 8, pp. 47664769, 1982.

[29] H. Reichert, P. J. Eng, H. Dosch, and I. K. Robinson, “Thermodynamics of surface segregation profiles at $\mathrm{Cu}_{3} \mathrm{Au}(001)$ resolved by x-ray scattering," Physical Review Letters, vol. 74, no. 11, pp. 2006-2009, 1995.

[30] S. P. Dash and H. D. Carstanjen, "Initial stages of growth of iron on silicon for spin injection through Schottky barrier," Physica Status Solidi B, vol. 248, p. 2300, 2011.

[31] K. Kimura, K. Ohshima, and M. H. Mannami, "Initial stage of Ag growth on $\mathrm{Si}(001)$ studied by high-resolution Rutherfordbackscattering spectroscopy," Physical Review B, vol. 52, no. 8, pp. 5737-5742, 1995.

[32] I. I. Pronin, M. V. Gomoyunova, S. M. Solov'ev, O. Y. Vilkov, and D. V. Vyalikh, "Initial stages of the growth and magnetic properties of cobalt films on the $\mathrm{Si}(100) 2 \times 1$ surface," Surface Physics, vol. 53, p. 616, 2011.

[33] V. F. Motsnyi, J. De Boeck, J. Das et al., "Electrical spin injection in a ferromagnet/tunnel barrier/semiconductor heterostructure," Applied Physics Letters, vol. 81, no. 2, p. 265, 2002.

[34] X. Jiang, R. Wang, R. M. Shelby et al., "Highly spin-polarized room-temperature tunnel injector for semiconductor spintronics using $\mathrm{MgO}(100)$," Physical Review Letters, vol. 94, no. 5, Article ID 056601, 4 pages, 2005.

[35] B. T. Jonker, G. Kioseoglou, A. T. Hanbicki, C. H. Li, and P. E. Thompson, "Electrical spin-injection into silicon from a ferromagnetic metal/tunnel barrier contact," Nature Physics, vol. 3, no. 8, pp. 542-546, 2007.

[36] O. M. J. van 't Erve, A. T. Hanbicki, M. Holub et al., "Electrical injection and detection of spin-polarized carriers in silicon in a lateral transport geometry," Applied Physics Letters, vol. 91, no. 21, Article ID 212109, 2007.

[37] T. Sasaki, T. Oikawa, T. Suzuki, M. Shiraishi, Y. Suzuki, and K. Tagami, "Electrical spin injection into silicon using $\mathrm{MgO}$ tunnel barrier," Applied Physics Express, vol. 2, no. 5, Article ID 053003, 2009.

[38] D. K. Fork, F. A. Ponce, J. C. Tramontana, and T. H. Geballe, "Epitaxial $\mathrm{MgO}$ on $\mathrm{Si}(001)$ for Y-Ba-Cu-O thin-film growth by pulsed laser deposition," Applied Physics Letters, vol. 58, no. 20, pp. 2294-2296, 1991.
[39] S. Mukhopadhyay and I. Das, "Inversion of magnetoresistance in magnetic tunnel junctions: Effect of pinhole nanocontacts," Physical Review Letters, vol. 96, no. 2, Article ID 026601, 4 pages, 2006.

[40] R. Wang, X. Jiang, R. M. Shelby et al., "Increase in spin injection efficiency of a CoFeMgO (100) tunnel spin injector with thermal annealing," Applied Physics Letters, vol. 86, no. 5, Article ID 052901, 3 pages, 2005.

[41] A. Fert and H. Jaffrès, "Conditions for efficient spin injection from a ferromagnetic metal into a semiconductor," Physical Review B, vol. 64, Article ID 184420, 2004.

[42] M. R. J. van Buuren, C. L. Griffiths, and H. van Kempen, "Chemical interactions and Schottky barrier determinations at the $\mathrm{Mg} / \mathrm{Si}(100)$ interface studied using X-ray photoelectron spectroscopy," Surface Science, vol. 314, no. 2, pp. 172-178, 1994.

[43] P. Hutchison, M. M. R. Evans, and J. Nogami, "Initial stages of Mg growth on the Si(001) surface studied by STM," Surface Science, vol. 411, no. 1-2, pp. 99-110, 1998.

[44] E. S. Cho, C. H. Lee, C. C. Hwang et al., "High-resolution corelevel photoelectron spectroscopy of $\mathrm{Mg} / \mathrm{Si}(100)$ surfaces," Surface Science, vol. 523, no. 1-2, pp. 30-36, 2003.

[45] G. J. van Gurp, W. F. van der Weg, and D. Sigurd, "Interactions in the $\mathrm{Co} / \mathrm{Si}$ thin-film system: II. Diffusion-marker experiments," Journal of Applied Physics, vol. 49, no. 7, pp. 40114020, 1978. 

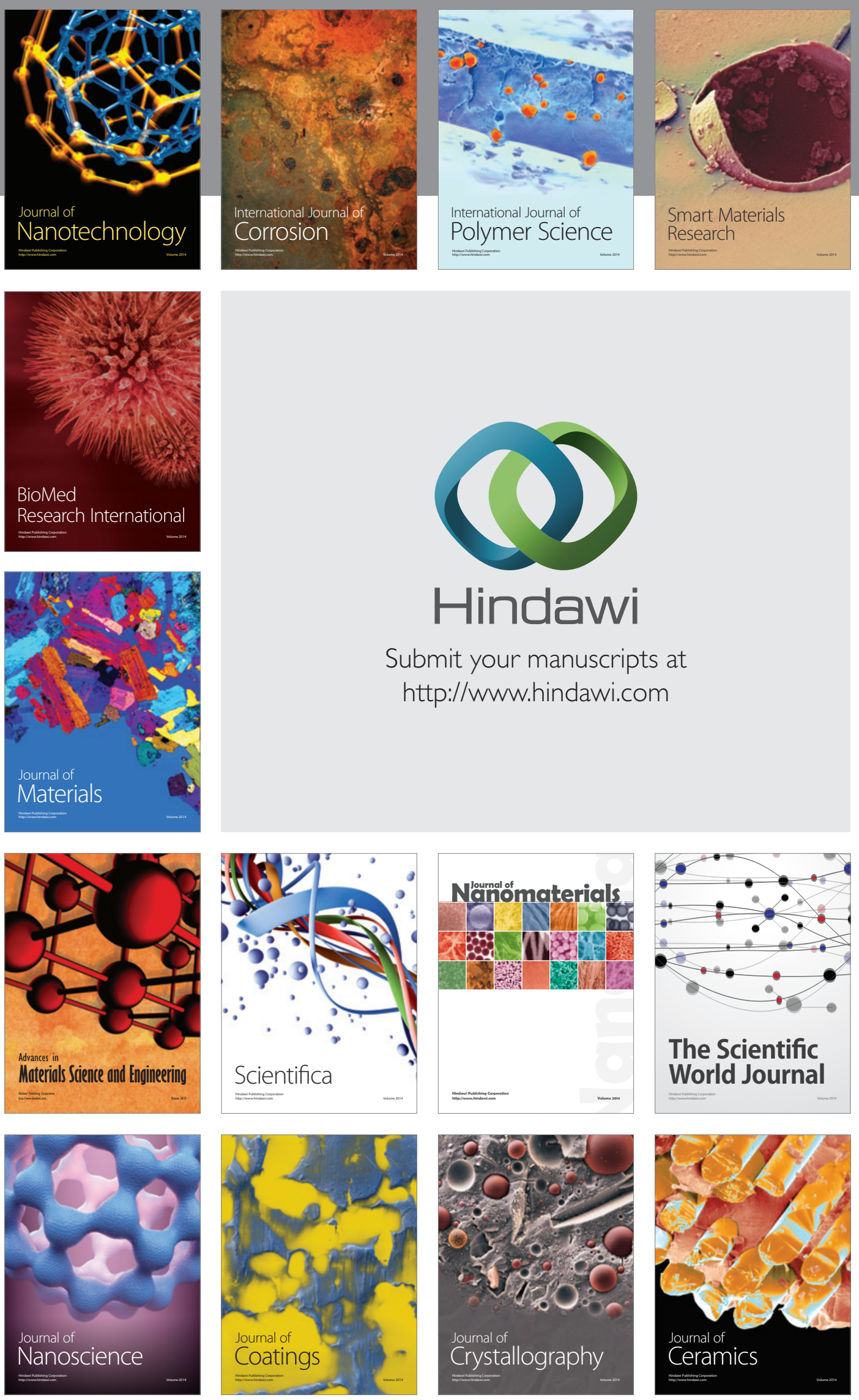

The Scientific World Journal

Submit your manuscripts at

http://www.hindawi.com

\section{World Journal}

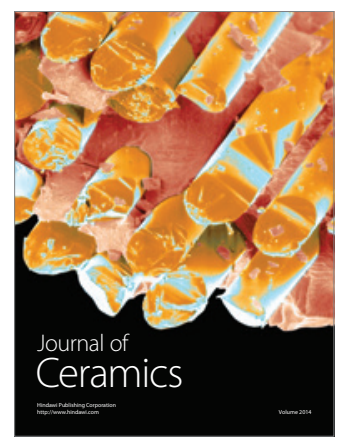

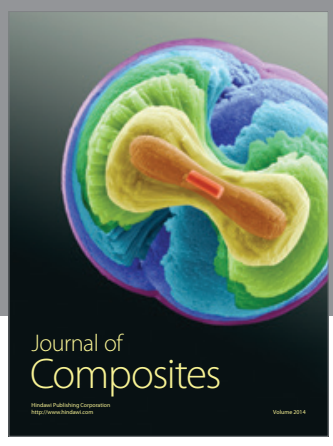
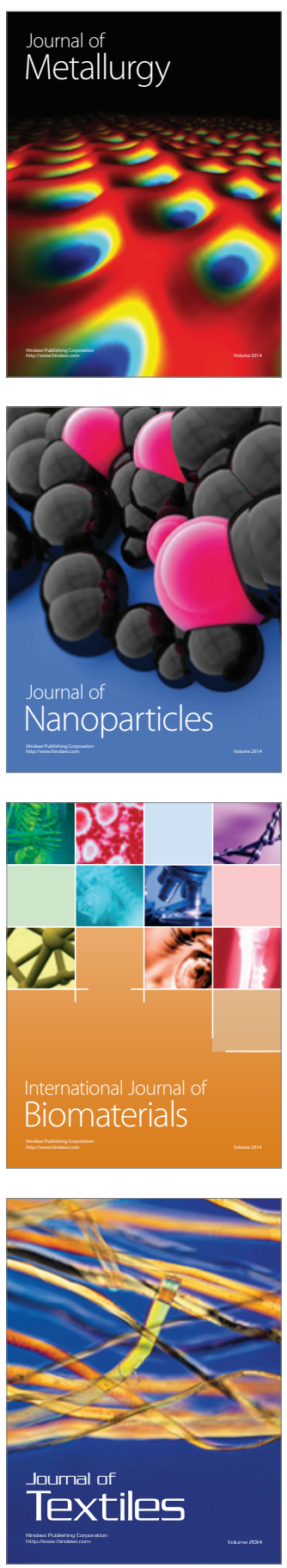